\title{
In ovo time-lapse analysis after dorsal neural tube ablation shows rerouting of
} \section{chick hindbrain neural crest}

\author{
Paul Kulesa*, Marianne Bronner-Fraser and Scott Fraser \\ Division of Biology and Beckman Institute, California Institute of Technology, Pasadena, CA 91125, USA \\ *e-mail: kulesa@gg.caltech.edu
}

Accepted 4 April; published on WWW 13 June 2000

\section{SUMMARY}

Previous analyses of single neural crest cell trajectories have suggested important roles for interactions between neural crest cells and the environment, and amongst neural crest cells. To test the relative contribution of intrinsic versus extrinsic information in guiding cells to their appropriate sites, we ablated subpopulations of premigratory chick hindbrain neural crest and followed the remaining neural crest cells over time using a new in ovo imaging technique. Neural crest cell migratory behaviors are dramatically different in ablated compared with unoperated embryos. Deviations from normal migration appear either shortly after cells emerge from the neural tube or en route to the branchial arches, areas where cell-cell interactions typically occur between neural crest cells in normal embryos. Unlike the persistent, directed trajectories in normal embryos, neural crest cells frequently change direction and move somewhat chaotically after ablation. In addition, the migration of neural crest cells in collective chains, commonly observed in normal embryos, was severely disrupted. Hindbrain neural crest cells have the capacity to reroute their migratory pathways and thus compensate for missing neural crest cells after ablation of neighboring populations. Because the alterations in neural crest cell migration are most dramatic in regions that would normally foster cellcell interactions, the trajectories reported here argue that cell-cell interactions have a key role in the shaping of the neural crest migration.

Key words: Neural crest, Hindbrain, Cell migration, In ovo, Ablation, Repatterning, Time-lapse imaging, Chick

\section{INTRODUCTION}

The cranial neural crest represents an excellent in vivo model system for the study of the mechanisms underlying cell motility and guidance. Neural crest cells undergo an epithelial to mesenchymal transition to form a migratory population of cells that leaves the dorsal neural tube in a cranial to caudal sequence and subsequently migrates along highly stereotyped routes that are characteristic of their axial level of origin. In the head region, neural crest cells migrate through an environment comprising extracellular matrix (ECM) and cranial mesenchyme. Some cranial neural crest cells subsequently differentiate into cranial sensory ganglia, whereas others enter the branchial arches and give rise to bones of the jaw. Their superficial migratory route just below the surface ectoderm renders them well suited for visualizing during early stages of migration as well as after entering the branchial arches. A major limitation in understanding the guidance of neural crest cell migration, however, has been the lack of an appropriate technique to observe neural crest cell migratory behaviors and cell trajectories in normal, as well as perturbed embryos.

The developing hindbrain, subdivided into eight segments called rhombomeres (Vaage, 1969; Lumdsen and Keynes, 1989), offers a morphological framework against which to analyze the migratory patterns of cranial neural crest cells. After emerging at the dorsal midline of the neural tube, these cells sort into three distinct streams that are separated by neural crest-free zones lateral to rhombomere (r) 3 and $\mathrm{r} 5$. The first stream of cells $(\mathrm{r} 1,2)$ populates the trigeminal ganglion and mandibular (first) branchial arch and is continuous with a large mass of cranial neural crest cells emigrating from the caudal forebrain and midbrain. The second stream (lateral to r4) populates the hyoid (second) branchial arch, as well as the geniculate and vestibular ganglia. The third stream $(\mathrm{r} 6,7)$ populates the third and fourth branchial arches and the superior, petrosal and nodose ganglia (Noden, 1975; D'Amico-Martel and Noden, 1983; Lumsden et al., 1991).

It remains unclear whether the guidance instructions for neural crest cells come from the neural tube prior to their emigration, from other emigrating neural crest cells or from the environment surrounding the neural tube. At one extreme, it seems possible that neural crest cells are prespecified to migrate to and form structures in a particular branchial arch (Noden, 1983). According to such a hypothesis, specification instructions would be conferred upon premigratory cells in the neural tube, perhaps based upon the rhombomere-specific patterns of Hox gene expression (Hunt et al., 1991; McGinnis and Krumlauf, 1992). Alternatively, neural crest cell streams might be sculpted by dynamic interactions with the neural tube, other neural crest cells and/or the environment. For example, some studies suggest that neural crest precursors might 
selectively die in $\mathrm{r} 3$ and $\mathrm{r} 5$ based on interactions with neighboring tissues (Lumsden et al., 1991; Graham et al., 1993). However, more recent experiments question the necessity of cell death and suggest instead that the microenvironment of the neural tube and/or adjacent mesenchyme may play the major role in patterning the neural crest cell streams (Farlie et al., 1999).

Time-lapse analyses of cranial neural crest cell migration in explanted chick embryos show a wide range of cell behaviors suggesting the operation of many different guidance mechanisms (Kulesa and Fraser, 1998, 2000). Some neural crest cells exit rapidly from the neural tube and migrate in a sustained direction. Other cells sort into a particular exiting stream more slowly, making frequent changes of direction or crossing between adjacent rhombomeres before leaving the neural tube. Neural crest cells often migrate by forming chain-like cell arrangements extending from the neural tube into the surrounding tissue. Time-lapse data reveal that cells avoid migrating into certain regions adjacent to the neural tube. For example, neural crest cells that enter the domain adjacent to $\mathrm{r} 3$ and $r 5$ either stop and collapse their filopodia or rapidly change direction to join a neighboring stream (Kulesa and Fraser, 1998). To examine the relative importance of different potential cues that might guide cranial neural crest migratory behavior, it is necessary to challenge and alter normal migratory behavior.

Efforts to disrupt the patterning of the branchial arches by ablating subpopulations of premigratory hindbrain neural crest cells have yielded embryos with surprisingly normal facial structures (McKee and Ferguson, 1984). For ablations done prior to stage 9 , embryos develop with normal face and neck morphology because the remaining hindbrain cells regulate to reform the neural crest (Scherson et al., 1993; Hunt et al., 1995; Sechrist et al., 1995; Buxton et al., 1997). When subpopulations of hindbrain neural crest cells are ablated after stage 9, embryos still develop with relatively normal head structures despite the fact that the regulative ability of the neural tube has declined by these later stages. This apparent normality results from neural crest cells rostral and/or caudal to the ablated neural tube deviating in their migration to 'fill in' for the ablated population (Couly et al., 1996; Hunt et al., 1998; Saldivar et al., 1997).

While it is clear that the embryo can compensate for the major ablation of the neural crest by at least two mechanisms, such endpoint analyses leave several basic questions about cell behaviors unanswered. What are the cell trajectories and migration behaviors of non-ablated neural crest cells in ablated embryos? Where and when do the cell trajectory deviations take place? To address such questions, we have performed in ovo time-lapse imaging studies of hindbrain neural crest migration. After dorsal neural tube ablation, DiI-labeled neural crest cells demonstrate profound alterations in their migratory behaviors and trajectories. Even cells arising significant distances from the ablated population appear to respond. Our results therefore suggest a large degree of intercommunication amongst hindbrain neural crest cells.

\section{MATERIALS AND METHODS}

\section{Embryos}

Fertile White Leghorn chick eggs were obtained from a local supplier
(Lakeview Farms) and incubated at $38^{\circ} \mathrm{C}$ until approximately the 611 somite stage (ss) of development. Eggs were rinsed with $70 \%$ ethanol and $3 \mathrm{ml}$ of albumin was removed prior to cutting a window through the shell. A solution of 10\% India ink (Pelikan Fount; PLK 51822A143) in Howard Ringer's solution was injected below the blastodisc to help visualize the embryos. Embryos were staged according to the criteria of Hamburger and Hamilton (1951) and/or by their number of somites, e.g. 10 somite stage (10 ss).

\section{In ovo preparation}

Eggs at 7-9 ss were windowed by cutting a hole in the egg shell. The vitelline membrane was deflected near the cranial region of the embryo using a fine tungsten needle. Embryos were injected with an isotonic sucrose solution of DiI (D-282; Molecular Probes) made from a $0.5 \%$ stock solution in $100 \%$ ethanol, diluted $1: 10$ in $0.3 \mathrm{M}$ warmed $\left(38^{\circ} \mathrm{C}\right)$ sucrose immediately before use. Dye solution was injected into the lumen of the hindbrain neural tube using a quartz micropipet (Sutter Instrument Co.) positioned just rostral to the first somite pair. A very small amount of Fast Green FCF (Fisher \#42053; $10 \mathrm{mg} / \mathrm{ml}$ ) was added to the DiI solution to aid in visualization during injection. Injected eggs were resealed with adhesive tape and incubated at $38^{\circ} \mathrm{C}$ for 1-2 hours. Each embryo was evaluated in terms of the brightness and uniformity of DiI labeling using an upright epifluorescence microscope (Zeiss) with a $10 \times$ long working distance (Nikon, LWD) objective and the best labeled embryos were selected. The DiI marked a large majority of neural crest cells and cells in the neural tube.

Bilateral ablations of the dorsal neural tube (termed 'ablation') were performed using a fine glass needle as described in Sechrist et al. (1995). Using the bulges and undulations of the hindbrain as landmarks for specific rhombomere borders, an incision was made between the ectoderm and neural tube followed by another incision that bisected the neural tube into dorsal and ventral halves. For removal of dorsal r5/6, a transverse cut was made rostrally (at the $\mathrm{r} 4 / 5$ border) and another one caudally (at the r6/7 border) to the otic vesicle to remove the dorsal third of the neural tube. Similarly, for r1-3 removals, a tranverse cut was made just rostral to $\mathrm{r} 1$ and just caudal to $\mathrm{r}$.

To allow in ovo imaging of both normal and operated embryos, a cylindrical acrylic ring $(2.2 \mathrm{~cm}$ inner diameter $\times 2.6 \mathrm{~cm}$ outer diameter $\times 0.5 \mathrm{~cm}$ high) was prepared as a window into the eggshell. The acrylic ring was held by forceps and one end was dipped into warmed white beeswax (Eastman Kodak Co. \#1126762) for approximately 10 seconds. The ring was then laid on a rectangular piece of $\mathrm{O}_{2}$ permeable membrane (Fisher \#13-298-83; $3.8 \mathrm{~cm} \times 7.5 \mathrm{~cm} \times 15 \mu \mathrm{m}$ ) and before the beeswax could harden, a rubber O-ring was placed around the circumference of the acrylic ring, pulling the teflon membrane taut around the sides of the ring. After 5 minutes, the rubber O-ring was removed and the excess teflon cut away. The teflon window was then positioned over the embryo, contacting the albumen and vitelline membrane. The ring was sealed to the eggshell with melted beeswax.

\section{Time-lapse video microscopy}

DiI-labeled embryos were visualized using an upright confocal microscope (Biorad MRC-500) modified with a multiple pole lowpass filter to better control the noise from the detector PMTs. The aperture of the confocal microscope was fully opened such that with a $10 \times$ Neofluor objective (NA 0.3 ) each optical section represented about $30-40 \mu \mathrm{m}$ in $z$-height. Although large aperature resulted in less confocal effect, it both increased the sensitivity of the microscope and allowed for a single optical section to capture a majority of the cell trajectories over longer periods of time. The microscope (Zeiss) was surrounded by an environmental chamber comprised of a cardboard box (4 mm thick) covered with thermal insulation (Reflectix Co.; 8 $\mathrm{mm}$ thick), and enclosing a warming heater (Lyon Electric Co. Inc., number 115-20). This maintained the temperature at $38^{\circ} \mathrm{C}$ for the duration of time-lapse filming, with only minor fluctuations. The 
fluorescent dye, DiI, was excited with the $568 \mathrm{~nm}$ laser line using the YHS filter set intended for rhodamine. Images were recorded every 2 minutes onto either a video optical memory disk recorder (OMDR; Panasonic TQ3038F) or digitally to magneto-optical disk (3M number $15175 ; 590 \mathrm{MB}$ ) using the COMOS software package. Images were analyzed and played back as a movie after conversion (by macros created by H. Karten, UCSD) using the image processing and analysis program, NIH Image 1.60.

\section{Time-lapse data analysis}

Time-lapse confocal images were analyzed with an automated 2-D cell tracking computer program (developed by Drs J. Solomon and S. Speicher, Caltech), which can follow a large number of labeled cells. The program identified cells from the initial image of the time series, and uses several criteria, including cell shape and brightness, to track the cells through the time-lapse sequence. A majority of the cells were tracked throughout the entire time-lapse sequence, with the program continuously calculating cell position and velocity. Some cells that were lost or suddenly appeared along the way were indexed by the program to be checked by the experimenter; many of these may result from cells moving in or out of the focal plane.

\section{RESULTS}

Premigratory neural crest cells were removed by ablation of the dorsal neural tube in subregions of the hindbrain. Migratory behavior of the neural crest cells emerging from the remaining hindbrain was visualized in ovo using timelapse imaging. Based on the major alterations in neural crest destinations after ablation of dorsal r5-6 (Saldivar et al., 1997), we initially analyzed r5-r6 ablations. To observe possible disruptions to neural crest cells migrating toward the first branchial arch (BA1) or BA2, we ablated dorsal r1-r3 or combinations of rhombomeres between r3-r6. All ablations were performed on 8-10 somite embryos, after which there is little regeneration of the remaining neural tube cells to reform neural crest (Sechrist et al., 1995). To test whether the normal neural crest cell migration pattern is disrupted due to the healing effects of the neural tube, we first injected DiI into the lumen of the neural tube and then ablated and replaced regions of the dorsal neural tube. When dorsal r5r6 $(n=8)$ and dorsal r1-r3 $(n=8)$ are ablated and replaced, time-lapse imaging shows that the neural crest cell migration pattern is similiar to that in normal intact embryos (data not shown). Cell tracking shows that the cells display velocities and directionalities indiscernible from controls. This suggests that the tissue response to healing has little effect on the migration pattern. Below, we describe the healing of the neural tube following ablation and analysis of the migratory behavior of neural crest cells adjacent to the ablated region.

\section{r7 neural crest cells deviate rostrally in r5-r6 ablations}

During the first 8 hours post-ablation, the dorsal neural tube heals and recloses. Immediately after the removal of the dorsal third of $\mathrm{r} 5$ and $\mathrm{r} 6$, a rectangular region (about $30 \mu \mathrm{m}$ wide by $200 \mu \mathrm{m}$ in length) of the dorsal neuroepithelium is clearly missing (Fig. 1A). Neural crest cells from rhombomeres adjacent to the site of ablation start to migrate. After 6 hours, the ablated site continues to close bringing the original cut surfaces into contact (Fig. 1B). By this time, the streams have formed adjacent to $\mathrm{r} 4$ and $\mathrm{r} 7$.

There are striking differences in the distribution of migrating hindbrain neural crest cells between unoperated (Fig. 2A,D) and operated embryos (Fig. 2B,E). Unoperated embryos show the distinct streams of neural crest cells laterally adjacent to $\mathrm{r} 1$, r2, r4 and r6, separated by neural crest free zones adjacent to r3 and r5 (Fig. 2A,D). In contrast, 6 hours after ablation the r6 stream is missing and a few cells are present lateral to r5 (Fig. $2 \mathrm{~B}, \mathrm{E})$. Interestingly, the neural crest cells lateral to $\mathrm{r} 7$ are further away from the neural tube (Fig. 2E). To highlight these changes, images of the unoperated and operated embryos have been colored red and green, respectively (Fig. 2C,F). Significant differences persist as the embryos develop further. Strikingly, r7 neural crest cells deviate rostrally toward the area
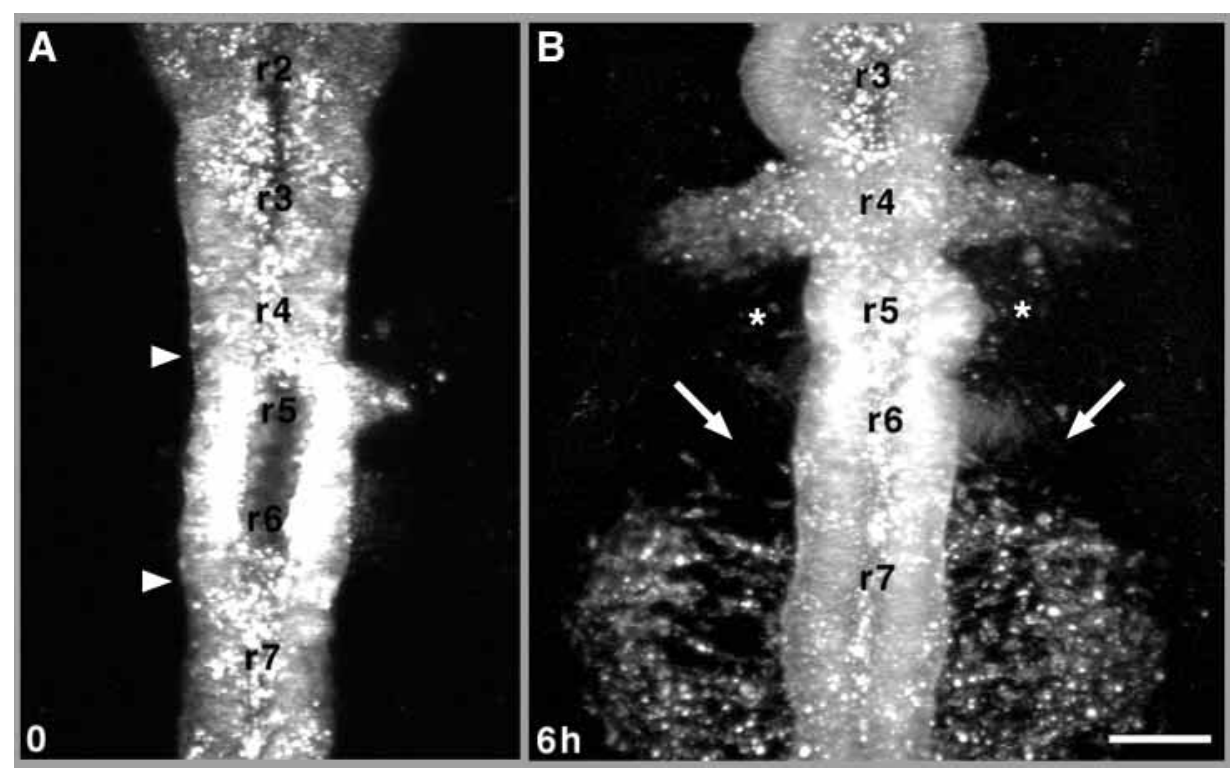

Fig. 1. Selected images from a time-lapse confocal imaging session showing the healing of the neural tube after the ablation of the dorsal portion of rhombomere 5 (r5) to r6. (A) DiI-labeled cells within the hindbrain are visible against the black background of the unlabeled tissue surrounding the neural tube. The arrowheads mark the rostral and caudal boundaries of the ablation site. This image was taken approximately 2 hours after the ablation. Notice that the walls of the neural tube have already started to close inwards. (B) After 6 hours (6h), neural crest cells have emigrated into the surrounding tissue. Notice that the typical, densely populated streams of cells that exit caudolaterally from adjacent to $\mathrm{r} 6$ and form the third branchial arch streams are absent (arrows). The region adjacent to $\mathrm{r} 3$ is neural crest cell free, but some nonablated cells have migrated to adjacent to $\mathrm{r} 5$ (asterisks). The distributions of neural crest cells adjacent to $\mathrm{r} 4$ and $\mathrm{r} 7$ appear as in normal embryos. The second branchial arch streams extend laterally from $\mathrm{r} 4$ and $\mathrm{r} 7$ cells have spread out to cover a wide region adjacent to $\mathrm{r} 7$. Bar, $100 \mu \mathrm{m}$. 


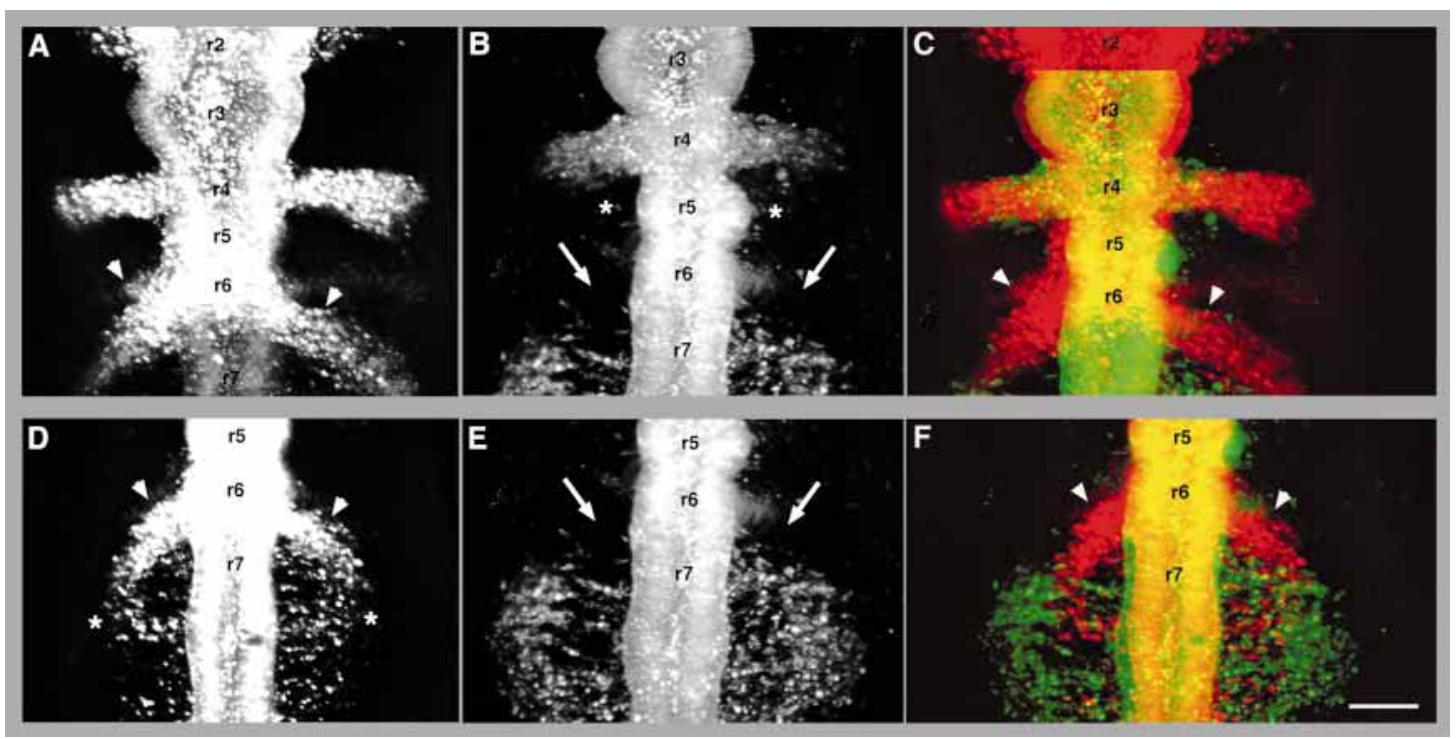

Fig. 2. Selected images from two distinct time-lapse sequences comparing the pattern of hindbrain neural crest cells in normal and dorsal r5-r6 ablated embryos. (A) In normal embryos, characteristic, densely populated streams of neural crest cells spread out into the tissue surrounding the neural tube adjacent to rhombomere 4 (r4) and r6. Notice how the streams exiting adjacent to r6 extend caudolaterally (arrowheads). Neural crest cell-free zones have been set up adjacent to r3 and r5. (B) In r5-r6 ablated embryos, the distribution of neural crest cells is dramatically different. In a representative r5-r6 ablated embryo, there are no densely populated neural crest streams emanating from r6 (arrows). However, neural crest cells have formed the second branchial arch streams extending from $\mathrm{r} 4$, and $\mathrm{r} 7$ neural crest cells have spread out to cover the region lateral to $\mathrm{r} 7$. The region adjacent to $\mathrm{r} 3$ is neural crest cell free, but some non-ablated cells have migrated to adjacent to r5 (asterisks). (C) The normal (red) and ablated (green) embryos shown in (A) and (B) are overlayed. Notice that the red streams exiting adjacent to r6 have very little or no overlap with green cells of the ablated embryo (arrowheads). The streams exiting adjacent to r4 overlap. (D-F) This set of images show the caudal region of the hindbrain in a similar comparison between normal and r5-r6 ablated embryos. (D) In a normal embryo, $\mathrm{r} 7 \mathrm{neural}$ crest cells spread out and distribute into the region lateral to $\mathrm{r} 7$ (asterisks). Notice that the streams adjacent to r6 extend caudolaterally (arrowheads) and overlap with the distribution r7-derived neural crest cells. (E) In r5-r6 ablations, the characteristic streams of neural crest cells exiting adjacent to r6 are noticeably absent (arrows); however, $r 7$ neural crest cells have spread out extensively into the region adjacent to the neural tube. (F) An overlay of the normal (red) and ablated (green) embryos shows that there is very little or no overlap of cells in the streams exiting from r6 (arrowheads). However, $r 7$ neural crest cells in the normal embryo are mixed in with $r 7$ neural crest cells of the ablated embryo. Notice that the $\mathrm{r} 7$ neural crest cells in the ablated embryo (green) have migrated laterally to beyond where the typical interaction with r6 neural crest cells would take place. Bar, $100 \mu \mathrm{m}$.
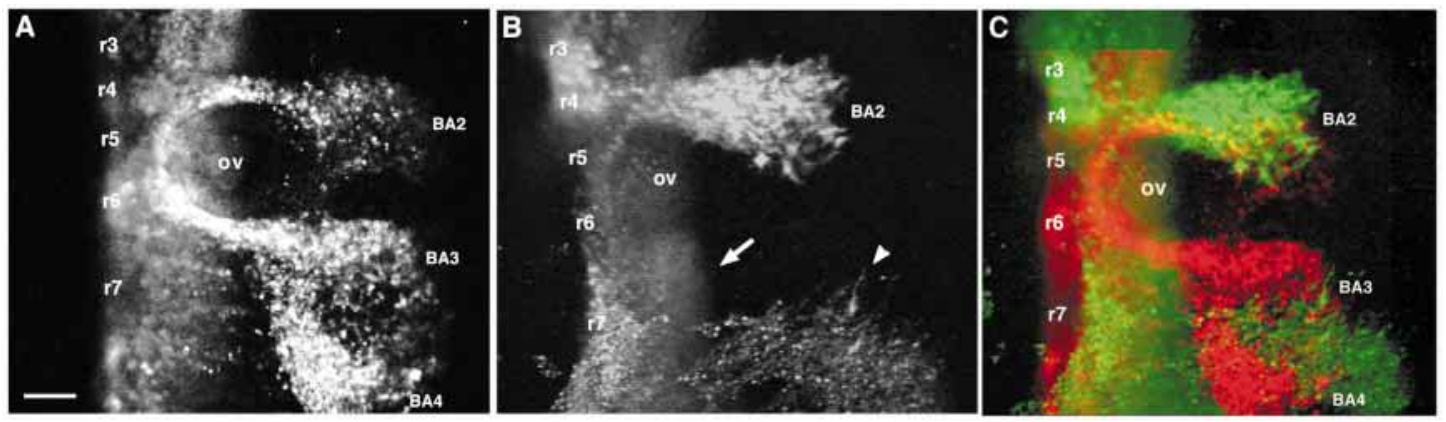

Fig. 3. Selected images from two distinct time-lapse sequences comparing the patterns of hindbrain neural crest cells in normal and r5-r6 ablated ablated embryos after the rotation of the embryo. These images show the right-hand side of the embryo, revealing the neural tube, otic vesicle (OV), second (BA2), third (BA3) and fourth (BA4) branchial arches. (A) In a representative normal embryo, the second branchial arch stream has migrated around the rostral portion of the OV and the front of the stream has expanded into BA2. The third branchial arch stream has extended from r6 in the caudolateral direction along the caudal part of the OV. This stream has split into two branches. The rostral portion of the stream extends to BA3 and the caudal part to BA4. (B) In the r5-r6 ablated embryos, the third branchial arch stream is completely missing (arrow). Notice how the neural crest cells, which are lateral to 7 , have turned and are migrating in the rostral direction. These cells are extending filopodia (arrowhead) in the rostral direction. The second branchial arch stream has extended towards BA2 and appears normal. (C) An overlay of the normal (red) and ablated (green) embryos shows that the second branchial arch streams overlap. Strikingly, the extent to which the missing third branchial arch stream in the ablated embryo is seen, as there is no overlap of red and green cells. Notice how the r7derived neural crest cells have migrated beyond the fourth branchial arch and deviated rostrally towards BA3. Bar, $100 \mu \mathrm{m}$. 


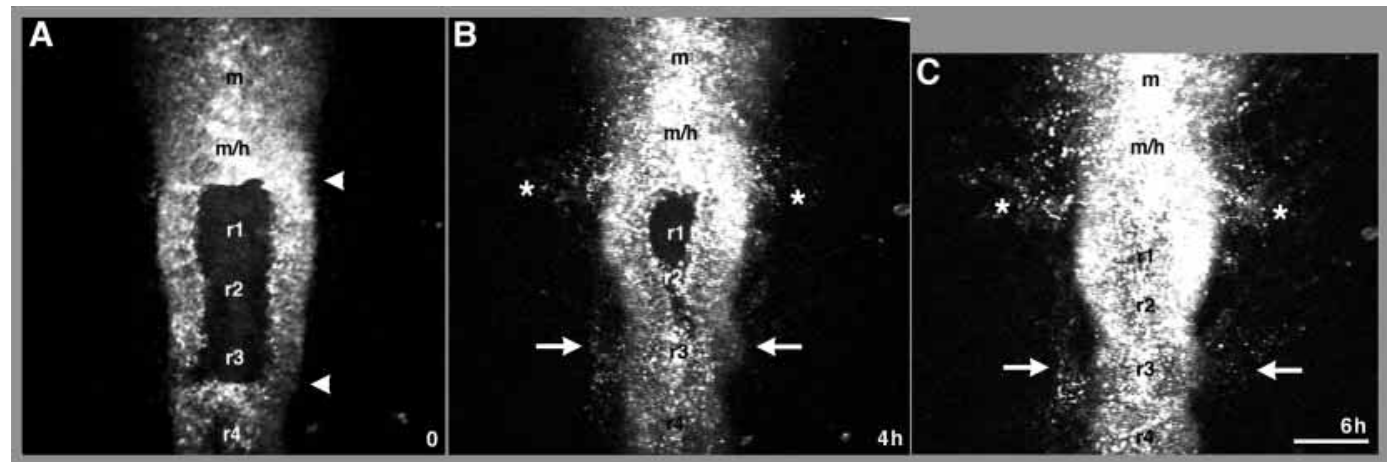

Fig. 4. Selected images from a time-lapse confocal imaging session showing the healing of the neural tube after the ablation of the dorsal portion of rhombomere 1 (r1) to r3. DiI-labeled cells within the hindbrain are visible against the black background of the unlabeled tissue surrounding the neural tube. (A) The arrowheads mark the rostral and caudal boundaries of the ablation site. This image was taken approximately 2 hours following the ablation. (B) After 4 hours (4h), the walls of the neural tube have started to close the ablated site. Neural crest cells have emigrated into the surrounding tissue. Notice that cells appear adjacent to the midbrain/hindbrain boundary $(\mathrm{m} / \mathrm{h})$ and rostral $\mathrm{r} 1$ (asterisks) and $\mathrm{r} 3$ (arrows). (C) By 6 hours (6h) more neural crest cells have filled into the region adjacent to rostral $\mathrm{r} 1$ (asterisks) and cells have also migrated into the area adjacent to $\mathrm{r} 3$ (arrows). The neural tube walls have mostly closed the ablated site. $\mathrm{m}$, midbrain. Bar, $100 \mu \mathrm{m}$.

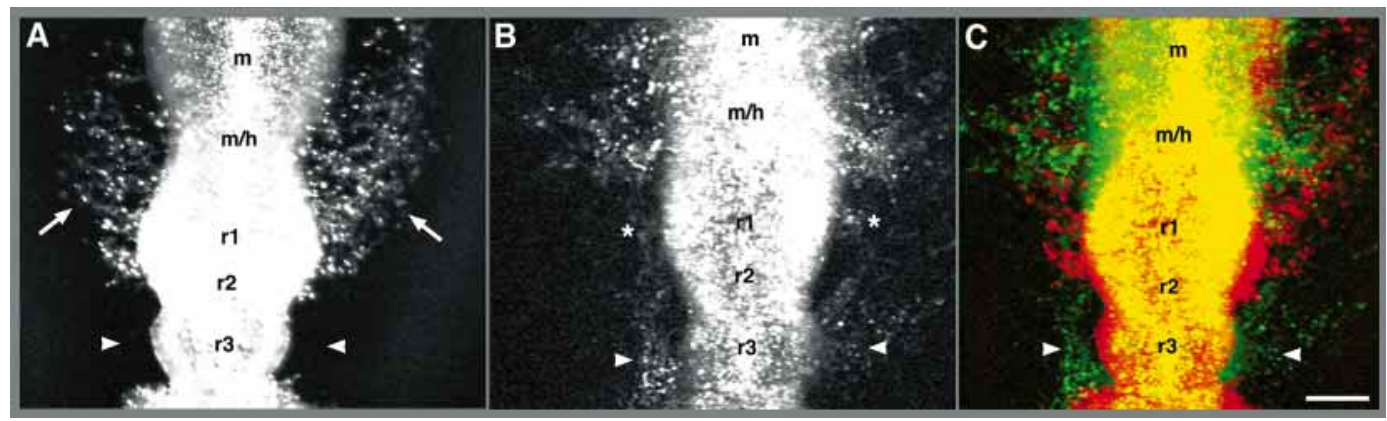

Fig. 5. Selected images from two distinct time-lapse sequences comparing the patterns of DiI-labeled hindbrain neural crest cells in normal and dorsal r1-r3 ablated embryos. (A) In a normal embryo, the characteristic pattern of neural crest cells emigrating from the midbrain and midbrain/hindbrain boundary $(\mathrm{m} / \mathrm{h})$ to $\mathrm{r} 2$ is spread out into the tissue surrounding the neural tube. The rostrolateral movements of the cells give rise to this typical pattern. The neural crest cell-free zones are adjacent to $r 3$ (arrowheads). (B) In r1-r3 ablated embryos, the distribution of neural crest cells is dramatically different. The regions adjacent to $\mathrm{r} 3$, which are typically neural crest cell-free zones, are populated with cells (arrowheads). Notice that the distribution of neural crest cells adjacent to the midbrain and the midbrain/hindbrain boundary ( $\mathrm{m} / \mathrm{h}$ ) is patchy and segregated, unlike the normal pattern, which is more uniform. Non-ablated neural crest cells have also filled in the region adjacent to rostral $\mathrm{r} 1$ (asterisks). (C) An overlay of the normal (red) and ablated (green) embryos from A and B shows that the areas adjacent to the midbrain and the $\mathrm{m} / \mathrm{h}$ have distributions of red and green cells which overlap, but the green cell distribution is patchy. The regions adjacent to caudal $\mathrm{r} 1$ and $\mathrm{r} 2$ show only red cells, reflecting the loss of ablated neural crest cells. The region adjacent to 3 has only green cells (arrowheads), revealing that non-ablated neural crest cells have moved into this typically neural crest cell free zone. Bar, $100 \mu \mathrm{m}$.

of the third branchial arch (Fig. 3B; an arrowhead points to extending filipodia), beyond the fourth branchial arch which they typically populate (Fig. 3C; unoperated, red; operated, green).

\section{Midbrain and $\mathbf{r} 4$ neural crest cells deviate to repopulate the regions adjacent to $\mathrm{r} 1$ and $\mathrm{r} 3$ in $\mathrm{r} 1-3$ ablations}

After ablating the dorsal one-third of the neural tube it heals much like after the r5-r6 ablations, with the remaining walls of the neural tube closing in on the ablated site in about 6 hours. Selected images from a time-lapse sequence show the ablated rectangle (about $50 \mu \mathrm{m}$ wide by $250 \mu \mathrm{m}$ in length) as it closes (Fig. 4) and as neural crest cells adjacent to the ablated site start to migrate (Fig. 4A-C). Although neural crest cells appear adjacent to the rostral half of r1 (Fig. 4B,C; asterisks) and lateral to r3 (Fig. 4B,C; arrows) there are very few cells in the region adjacent to caudal $\mathrm{r} 1$ and $\mathrm{r} 2$. A comparison of the pattern of neural crest cell migration in unoperated and r1-r3 ablated embryos is shown in Fig. 5. Unlike the normal broad domain of neural crest cells adjacent to the midbrain/hindbrain boundary and r1/r2 (Fig. 5A; arrows), neural crest cells in r1r3 ablated embryos distribute in a patchy arrangement adjacent to the neural tube (Fig. 5B). Furthermore, after ablation, neural crest cells are present in the regions adjacent to r3 (Fig. 5B; arrowheads), unlike the unoperated control. An overlay of unoperated (red) and r1-r3 ablated (green) images show the extent of these differences.

\section{Neighboring neural crest cells fill in the second branchial arch stream in r3-r4 and r4+r6 ablations}

To deplete the second branchial arch neural population, we 
Fig. 6. Selected images from a timelapse confocal imaging session showing cell movements after the ablation of the dorsal portion of rhombomere 3 (r3) to r4. DiI-labeled cells are visible against the black background of the unlabeled tissue surrounding the neural tube.

(A) Some individual neural crest cells are circled to make it easier to follow their migration pathways. The orange, yellow, green and red cells have emerged from $\mathrm{r} 5$ and are near the lateral portion of r5. The blue circled cell has emerged from near the r5/r6 boundary. (B) The orange circled cell has emigrated into a stream exiting adjacent to the $\mathrm{r} 4 / \mathrm{r} 5$ boundary. The yellow, green and red circled cells are also moving in this direction. The magenta circled cell is now visually distinguishable near the r5 midline. (C) As the orange circled cell continues to move away from the neural tube lateral to $r 4$, the magenta, yellow and green circled cells are also moving to join this exiting stream. The red and blue circled cells have moved to the lateral extent of r5. (D) All of the circled cells have joined the stream exiting adjacent to $r$, with the exception of the blue colored cell which has migrated to join the stream exiting adjacent to r6. Bar, $100 \mu \mathrm{m}$.
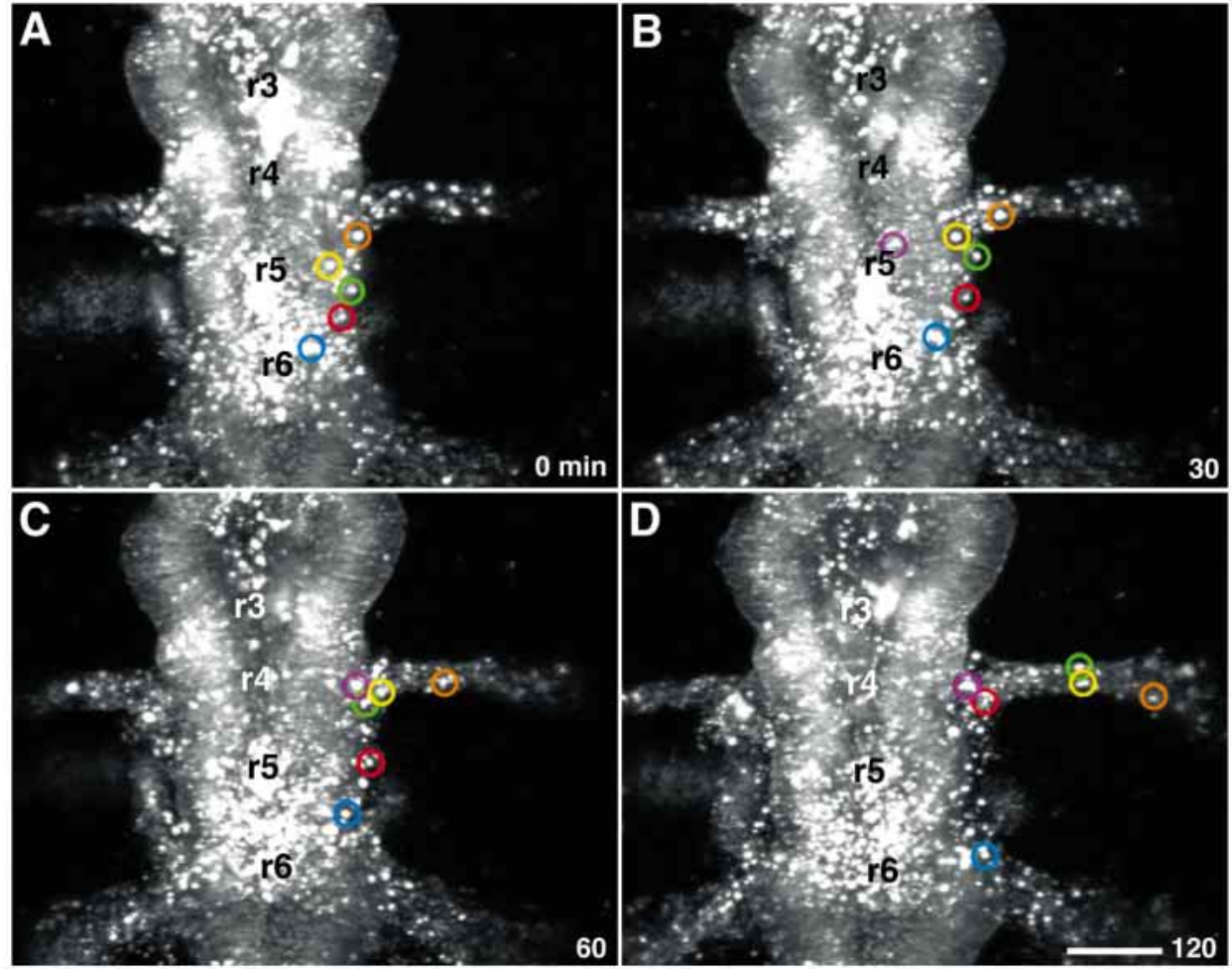

Fig. 7. Selected images from two distinct time-lapse sequences comparing the cell trajectories of migrating neural crest cells in normal and r5-r6 ablated embryos. Only a portion of the cell trajectories are shown for clarity, plotted against the background of the DiI-labeled neural tube (the black/white color table has been inverted so that labeled cells appear black). (A) In the normal embryo, notice how many of the cell trajectories extend in the lateral direction from caudal r6 and r7. Cell trajectories in the region where $r 6$ and $r 7$ neural crest cells interact adjacent to the neural tube are more twisted and turned, reflecting changes in cell direction. (B) In the r5r6 ablated embryos, cell trajectories are strikingly different, with many twists and turns. The cell trajectories in this representative embryo reflect the chaotic migratory

behavior of $\mathrm{r} 7$ neural crest cells which are observed in time-lapse imaging. Notice that some neural crest cells migrate in the rostral direction towards r5. Along the lateral edge of the neural tube, cell trajectories overlap with many cell changes in direction and very little lateral progression. Farther from the neural tube, a few cell trajectories have more sustained lateral movements. A sample of some typical cell trajectories from A and B highlight the dramatic difference in migratory pathways of cells emerging from r7 in normal (C) and r5-r6 ablated (D) embryos. Bar, $100 \mu \mathrm{m}$.

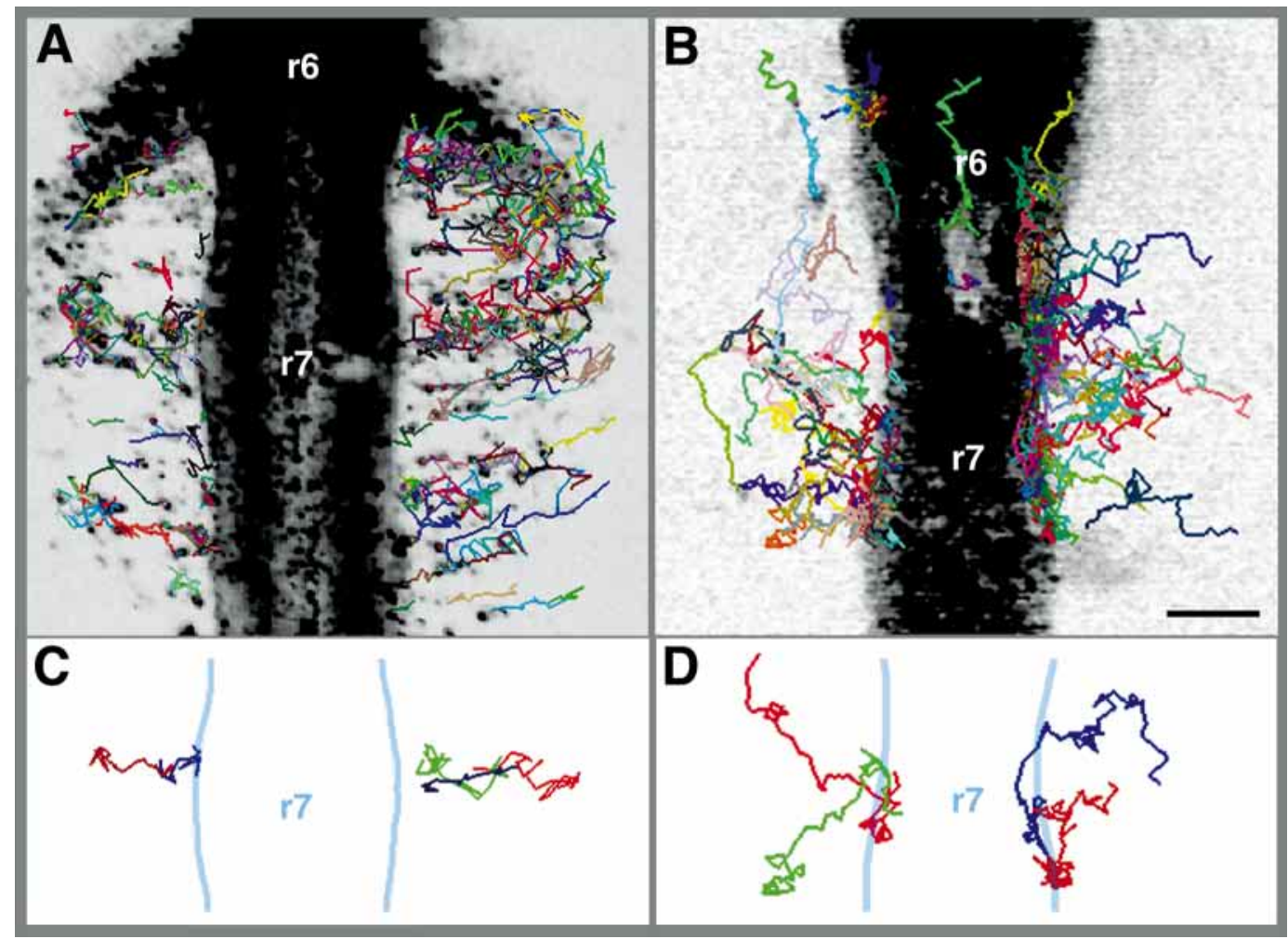


performed ablations that removed dorsal $\mathrm{r} 4$ in combination with either more rostral or caudal rhombomere(s), and examined the subsequent behavior of neighboring neural crest cells. In normal embryos, r3, r4 and r5 neural crest cells form the second branchial arch stream and populate the second branchial arch (Birgbauer et al., 1995; Kulesa and Fraser, 2000). Regardless of the paired combinations of rhombomeres between and including r3 to r6, non-ablated neural crest cells managed to fill in the second branchial arch stream. For example, following r3-r4 ablations, neural crest cells from r5 migrate into the second branchial arch (Fig. 6). Many neural crest cells from caudal $\mathrm{r} 5$, which otherwise would have been expected to invade the third branchial arch, apparently reroute their migratory pathway to join the second branchial arch stream (dark green and red circled cells in Fig. 6).

Ablations of dorsal $\mathrm{r} 4$ and $\mathrm{r} 6$ in the same embryo result in a decrease in the numbers of neural crest cells in both the second and third branchial arches. Neural crest cells from r3 and r5 populate the second branchial arch. Interestingly, the various pairs of ablations that eliminated $\mathrm{r} 4$ neural crest cells showed that the $\mathrm{r} 3$ population migrates within the rostral portion of the second branchial arch, whereas the r5 population predominantly occupies the caudal portion of the second branchial arch.

\section{Neural crest cell trajectories become less ordered in ablated embryos}

By employing an automated cell tracking program, the detailed trajectories of the neural crest cells can be revealed and show that ablated embryos display very different migratory behaviors. Under normal circumstances, neural crest cells emerge from the dorsal midline of the neural tube, sort into exit streams, and migrate toward the branchial arches. Cells migrating within exiting streams generally maintain a uniform direction toward a particular

Fig. 8. Selected images from two distinct time-lapse sequences comparing the vector representation of cell trajectories of migrating neural crest cells in normal and r5-r6 ablated embryos. (A,C) Only a portion of the cell trajectories are shown for clarity, plotted against the background of the DiI-labeled neural tube. Each vector is generated by constructing a straight line from the start to end (arrowhead) positions of individual cell trajectories over a 6 hour period. (B,D) Polar histograms were generated by measuring the angle that each vector makes with the horizontal (the horizontal is constructed at a $90^{\circ}$ angle to the rostrocaudal axis of the embryo). Vectors with angle measures greater than $90^{\circ}$ but less than $270^{\circ}$ are reflected across the rostrocaudal axis of the embryo so that all angle measures lie in the range of $-90^{\circ}\left(270^{\circ}\right)$ to $90^{\circ}$. The polar histograms are plotted in $15^{\circ}$ bins. (A) In normal embryos, notice how most vectors of $\mathrm{r} 7$ neural crest cells point in the lateral direction. (B) This is reflected in a majority of the vectors having angle measures from $0^{\circ}$ to $30^{\circ}$. (C) In r5r6 ablated embryos, the chaotic cell migratory behaviors give rise to cell trajectories that have vector

representations pointing in many directions. (D) Notice that the histogram shows the wide range of vector directions of cell migration in r5-r6 ablated embryos. Bars, $100 \mu \mathrm{m}$. branchial arch with only slight deviations, moving both as individuals and in collective chain-like cell arrangements (Kulesa and Fraser, 1998; Fig. 7A). In contrast, neural crest cell migratory behaviors in ablated embryos are more unpredictable, with the majority of cells displaying frequent changes in direction and/or sustained movement along an abnormal migration pathway. After r5-r6 ablations, r7 neural crest cells near the neural tube display chaotic migratory behaviors (Fig. 7B), frequently change directions and fail to make much lateral progress. Cell trajectories away from the neural tube are more sustained, but occur in a wide variety of directions (Fig. 7B).

The abnormal trajectories do not result from an inability of the cells to migrate, but from an error in the guidance of the migration. The average cell speeds of $173 \pm 5 \mu \mathrm{m} /$ hour (in ablated embryos) versus $166 \pm 4 \mu \mathrm{m} /$ hour (in unoperated embryos) are similar. The alterations in the migration of neural crest cells after ablation is easily seen in a plot in which each cell trajectory is represented as a vector from its starting (tail) and ending (head; arrowhead) positions over 6 hour time-lapse sessions. In unoperated embryos, most of the vectors point lateral from the neural tube (Fig. 8A), as shown by the polar histogram of the angle of the vectors (Fig. 8B). The majority of the $\mathrm{r} 7$ neural crest cells $(61 \%)$ migrate in a $\pm 30^{\circ}$ angle from the horizontal. In r5-r6 ablated embryos, the cell trajectory
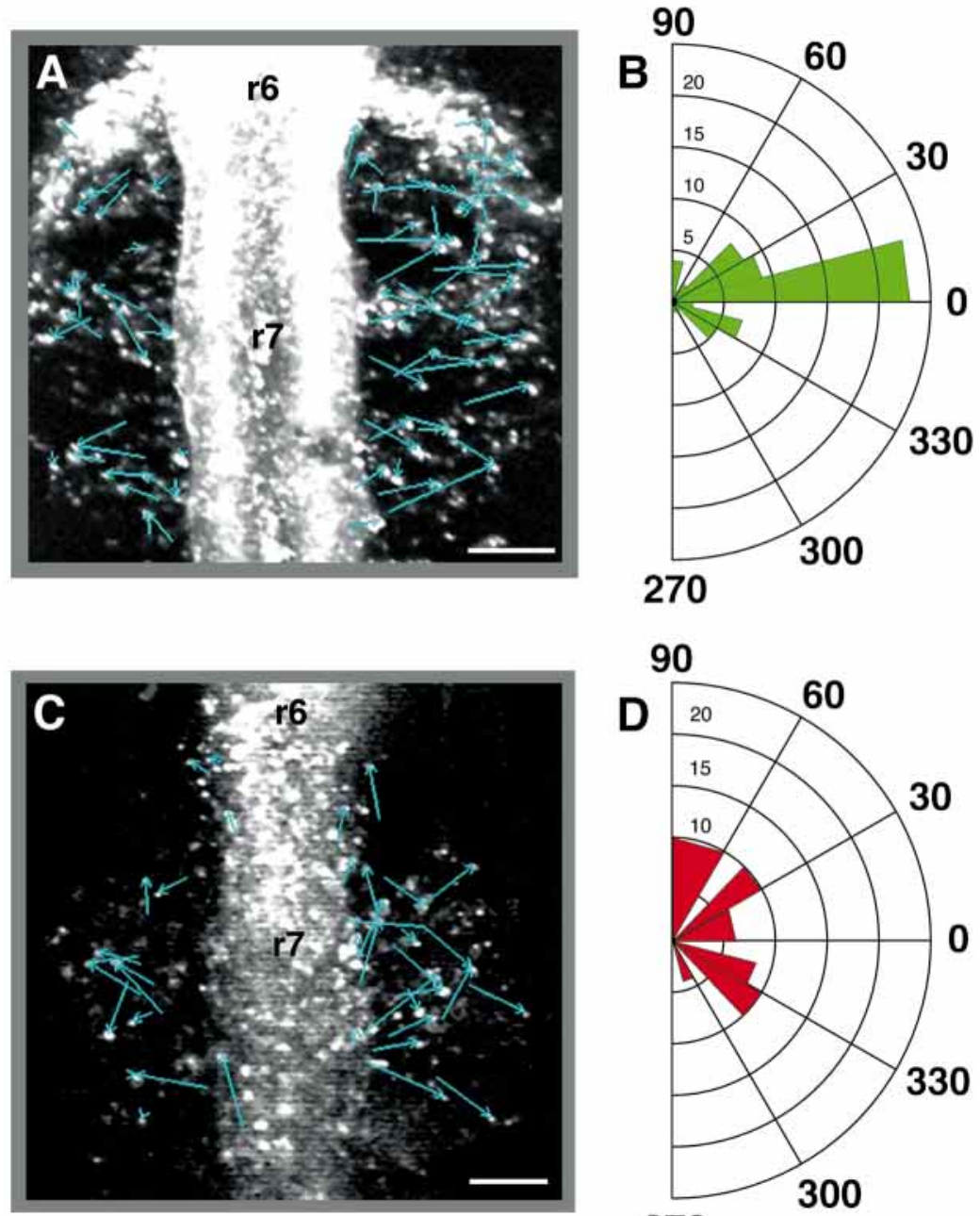

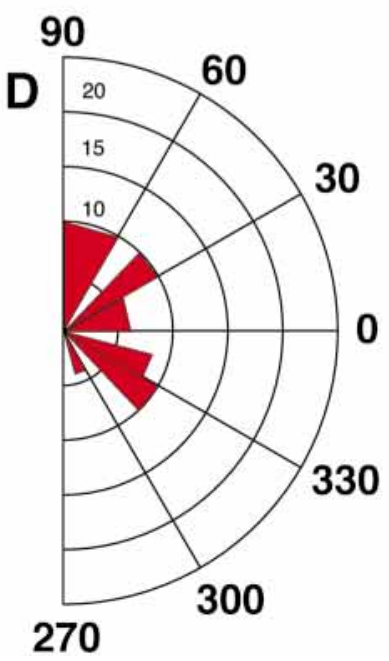




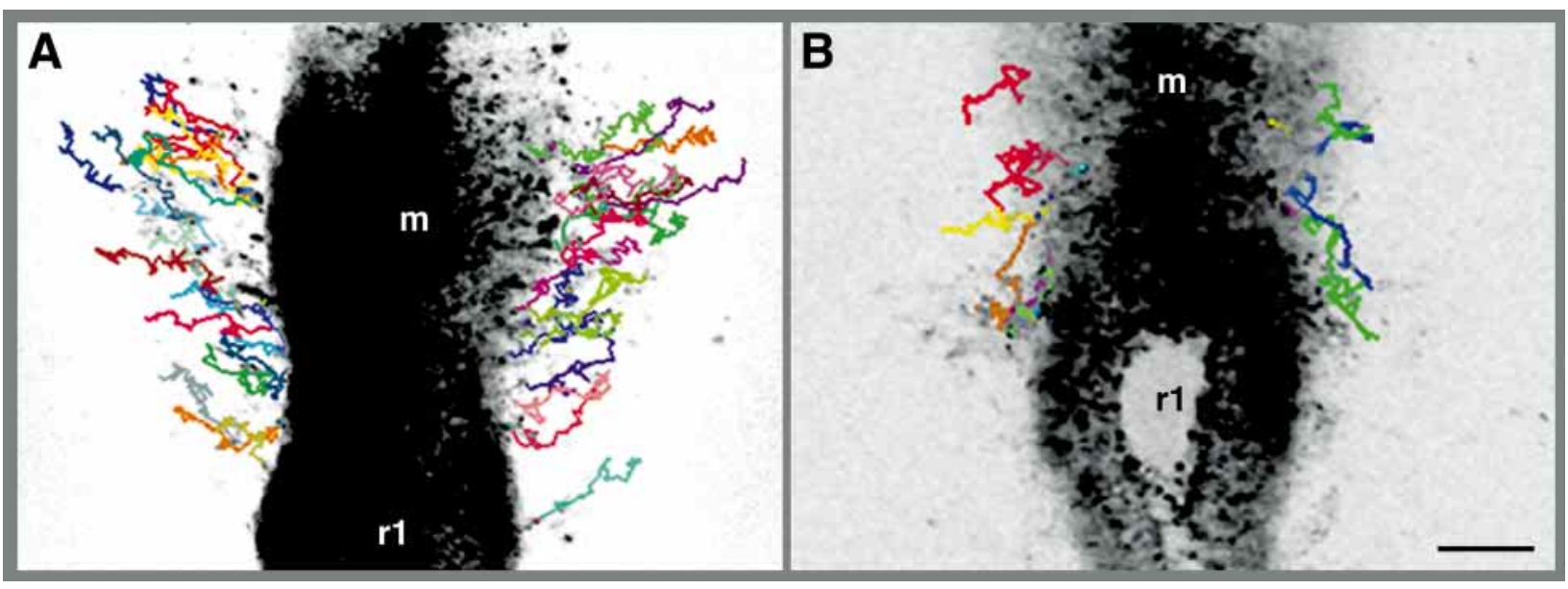

Fig. 9. Selected images from two distinct time-lapse sequences comparing the cell trajectories of migrating neural crest cells in normal and r1r3 ablated embryos. Only a portion of the cell trajectories are shown for clarity, plotted against the background of the DiI-labeled neural tube (the black/white color table has been inverted so that labeled cells appear black). (A) In the normal embryo, notice how many of the cell trajectories extend in the rostrolateral direction as cells migrating from the midbrain $(\mathrm{m})$ to $\mathrm{r} 1$ move towards the first branchial arch. The cell trajectories show that many cells maintain migration in a sustained direction. (B) In r1-r3 ablated embryos, notice that several cell trajectories lead in the caudolateral direction away from the direction of the first branchial arch. Bar, $100 \mu \mathrm{m}$.

vectors are more varied (Fig. 8C) and the angles are distributed over a much wider range (Fig. 8D). Only $30 \%$ of the cell trajectory angles lie in the $\pm 30^{\circ}$ angle range (Fig. $8 \mathrm{D}$ ).

In dorsal r5-r6 ablated embryos, we did not see $\mathrm{r} 7$ neural crest cells migrating in the chain-like cell arrangements that are typically observed in unoperated embryos. Instead, cells tended to migrate almost exclusively as individuals. In normal embryos, the chains of cells form near the lateral edge of the neural tube where cells organize into a linear array, which propagates in a lateral direction. Cells emerging from the dorsal midline join the collective by filling in at the medial side of

Fig. 10. Selected images from two distinct time-lapse sequences comparing the vector representation of cell trajectories of migrating neural crest cells in normal and r1-r3 ablated embryos. (A,C) Only a portion of the cell trajectories are shown for clarity, plotted against the background of the DiI-labeled neural tube. Each vector is generated by constructing a straight line from the beginning to end positions of individual cell trajectories over a 6 hour (6h) period. (B,D) Polar histograms were generated by measuring the angle that each vector makes with the horizontal (the horizontal is constructed at a $90^{\circ}$ angle to the rostrocaudal axis of the embryo). Vectors with angle measures greater than $90^{\circ}$ but less than $270^{\circ}$ are reflected across the rostrocaudal axis so that all angle measures lie from $-90^{\circ}\left(270^{\circ}\right)$ to $90^{\circ}$. The polar histograms are plotted in $15^{\circ}$ bins. (A) In normal embryos, notice how most vectors of midbrain and r1 neural crest cells point in the rostrolateral direction. (B) This translates into a majority of the vectors having angle measures from $0^{\circ}$ to $60^{\circ}$. (C) In r1-r3 ablated embryos, the change in cell movements to fill in adjacent to $\mathrm{r} 1$ and $\mathrm{r} 3$ shows that cell trajectories have vector representations pointing in caudolateral direction. (D) Notice that the histogram reflects the wide diversity of vector directions of cell trajectories. Several cells migrate in the direction between $0^{\circ}$ and $-90^{\circ}\left(270^{\circ}\right)$. Bar, $100 \mu \mathrm{m}$. the chain as it moves towards the branchial arches. Some neural crest cells that emigrate as individuals eventually join an existing chain.

In r1-r3 ablated embryos, cell migration behaviors are less chaotic than in r5-r6 ablations, but neural crest cells from neighboring regions still divert their trajectories and migrate
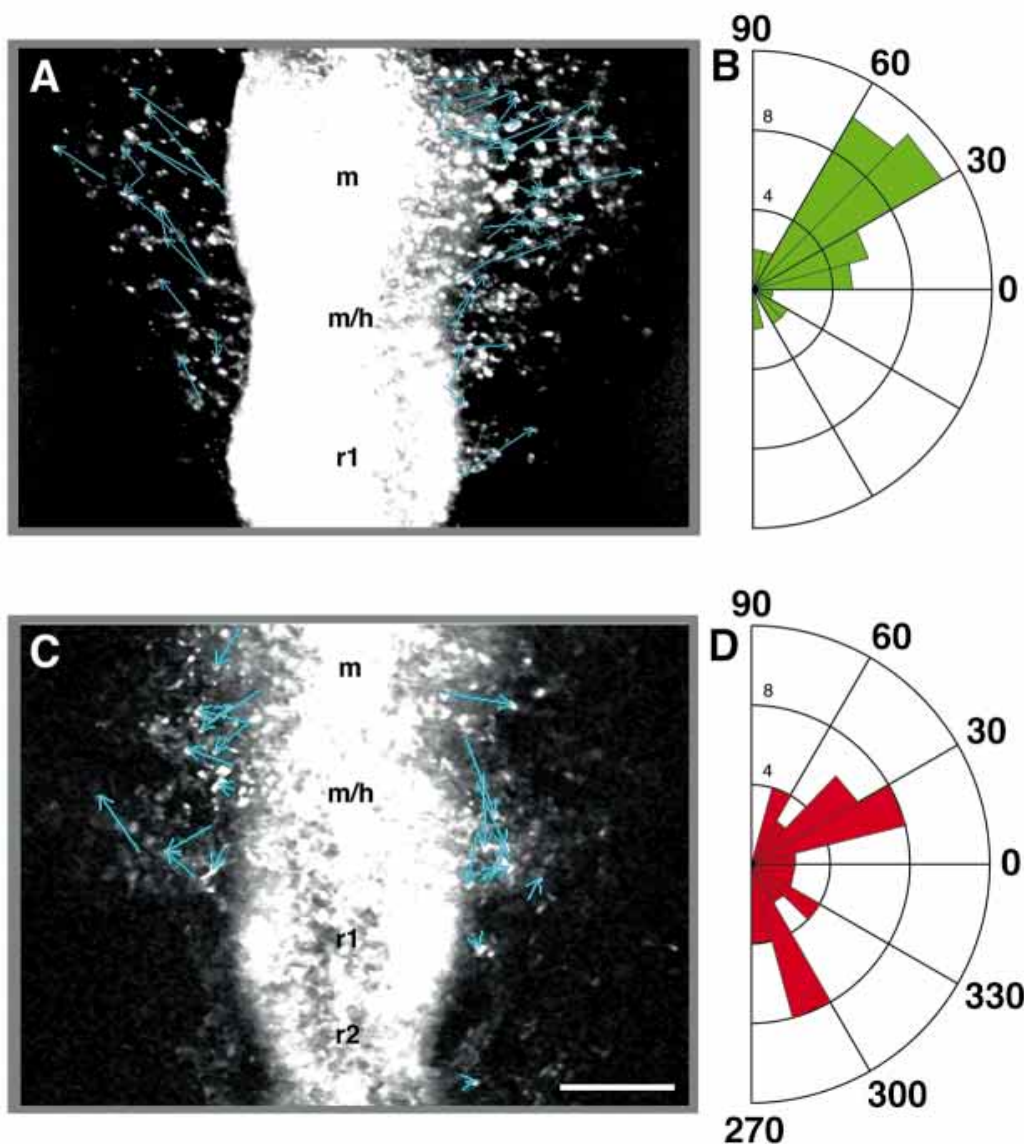
toward regions adjacent to the ablated areas. In unoperated embryos, neural crest cells from the midbrain to r1 spread out in a wide stream along trajectories that maintain a sustained direction, giving rise to a uniform distribution of cells adjacent to the neural tube (Fig. 9A). In r1-r3 ablated embryos, neural crest cells from the midbrain and midbrain/hindbrain boundary areas frequently change directions or move in a sustained progression in the caudolateral direction (away from the first branchial arch) into the region adjacent to r1 (Fig. 9B). The changes in cell trajectory direction take place as cells enter the tissue surrounding the neural tube. Average cell speeds of $147 \pm 3 \mu \mathrm{m} /$ hour (ablated embryos) versus $158 \pm 2 \mu \mathrm{m} /$ hour (unoperated embryos) are similar.

Cell trajectories in $\mathrm{r} 1-\mathrm{r} 3$ ablations show a wide distribution of angles. Fig. 10 shows a comparison between cell trajectories in representative operated and unoperated embryos, including the vector representations of the cell trajectories over a 6 hour period in typical time-lapse sessions. In unoperated embryos, many of the cell trajectory vectors point in the rostrolateral direction (Fig. 10A). In these embryos, nearly $70 \%$ of the neural crest cells from the midbrain to 1 migrate in a direction between $0^{\circ}$ and $60^{\circ}$, measured from the horizontal angle with the embryonic axis (Fig. 10B). In r1-r3 ablated embryos, the cell trajectory vectors point in many different directions (Fig. $10 \mathrm{C})$. There is a wide distribution of cell trajectory angles with as many as $50 \%$ of the cells migrating in the direction of $0^{\circ}$ to $-90^{\circ}$ reflecting the deviation in direction from the midbrain towards the region adjacent to $\mathrm{r} 1$ (Fig. 10D).

\section{DISCUSSION}

Both intrinsic and extrinsic cues have been implicated in guiding cell migration, ranging from cell-dependent cues such as population pressure (LeDouarin, 1982), to environmental cues such as contact guidance (Weiss, 1961) or adhesive gradients in the environment (Carter, 1967). Cranial neural crest cells are an excellent system for in vivo studies of cell movement because their superficial position in the embryo makes them accessible to observation during most of their extensive migration. To date, however, little is known about the mechanisms guiding their movement in vivo. In fact, most of what is known about cell migration is derived from analysis of cell behavior in vitro. The mechanisms guiding embryonic cells through the three-dimensional environment of the embryo may differ from those evident in planar tissue culture conditions. For example, neural crest cells in three-dimensional gel cultures appear somewhat different from migrating fibroblast cells (Bilozur and Hay, 1988) in that they do not possess a ruffled membrane in their leading filopodium and do not leave behind their trailing edge. Such differences provide the incentive to develop culture and imaging techniques to study cell migration in vivo in both normal and experimentally manipulated embryos. In our previous imaging studies of explanted chicken embryos (Kulesa and Fraser, 1998) or embryos in ovo (Kulesa and Fraser, 2000), a diverse array of cell behaviors was observed, implicating a variety of cell-cell and cell-environmental interactions in shaping neural crest cell migration.

In this paper, we explore the migratory properties of neural crest cells by combining experimental perturbations (surgical ablations) with a new in ovo imaging technique that makes it possible to follow cell trajectories over time (Kulesa and Fraser, 2000). After ablation of the dorsal neural tube, cranial neural crest cells have a remarkable ability to regulate and eventually populate the branchial arches, as if no ablation was performed. Depending upon the time of ablation, this occurs either by the regeneration of the neural tube to form neural crest or by the neural crest cells rostral or caudal to the ablated area filling in regions devoid of neural crest (Scherson et al., 1993; Sechrist et al., 1995; Hunt et al., 1995; Kirby et al., 1997; Grapin-Botton et al., 1995; Saldivar et al., 1997). By ablating the dorsal neural tube after the regenerative phase, we are able to monitor the filling-in process. This offers a test of the relative contribution of intrinsic versus extrinsic information in guiding hindbrain neural crest cells to their appropriate sites. The trajectories of individual neural crest cells make it clear that few if any cells continue to migrate to their normal destinations within branchial arches. For example, after r1-r3 ablations, many neural crest cells from the caudal midbrain alter their path and fill in the region adjacent to rostral $\mathrm{rl}$ (Fig. 4B,C; asterisks). These cells detour in a caudolateral direction shortly after entering the tissue surrounding the neural tube (Figs 9B, 10C). Analogously, r4 neural crest cells, instead of continuing in a lateral direction after leaving the neural tube, divert rostrally and move into the region adjacent to $\mathrm{r} 3$ (Fig. 4C; arrows). This change in behaviors would not be predicted if these cells were prespecified to migrate to defined destinations or if cell-environment interactions were the sole guidance mechanism. Instead, the data support the idea that cell-cell and cell-environment interactions play key roles in guiding neural crest cells.

The regions in which 're-routing' occurs are intriguing because they correlate with areas where typical cell-cell interactions take place between neural crest subpopulations from neighboring rhombomeres. In normal embryos, r6 and r7 neural crest cells migrate to the same spatial location in the fourth branchial arch (BA4) but their migratory streams mix only upon reaching the branchial arch (Kulesa and Fraser, 2000). In r5-r6 ablated embryos in which the r6-derived neural crest cell stream is eliminated, $r 7$ neural crest cells emerge and migrate in a chaotic, disorganized manner (Fig. 7B). Cells often change directions and fail to progress in a sustained manner. Eventually, r7-derived neural crest cells spread laterally towards BA4, but then migrate beyond where they typically interact with r6-derived neural crest cells and turn rostrally at near right angles and move towards the third branchial arch (Fig. 3B). In r1-r3 ablated embryos, neural crest cells from the midbrain region change their migration pathways soon after entering the tissue adjacent to the neural tube (Fig. 9B); this suggests that the absence of interaction with r1 neural crest and/or the local environment may play a role in redirecting cells into the first branchial arch.

The rerouting of neural crest cells appears to involve a significant change in the nature of the cell-cell interactions. For example, in ablated embryos the cells rarely migrate in 'chains' that are typical of unperturbed embryos (Kulesa and Fraser, 1998, 2000). In unoperated embryos, chains form as cells emerge at the dorsal midline and organize into a linear array that propagates in a lateral direction. This loss of chain migration may, at least in part, account for the significant difference in the migration pathways: in r5-r6 ablated embryos, 
neural crest cells migrate chaotically, often changing directions and moving almost exclusively as individuals. In r1-r3 ablations, cell migratory behaviors are less chaotic; however, many individual midbrain cells migrate along the lateral edge of the neural tube in the region where chain-like arrangements typically form.

Our time-lapse analyses of in ovo hindbrain neural crest cell migration paints a detailed picture of the repatterning of migration pathways after ablations. Cell trajectories show that both individual and collective cell migratory behaviors are dramatically altered, resulting in a rerouting of pathways to the branchial arches. The remaining cells often move chaotically, frequently change directions, and fail to organize into the collective chain-like cell arrangements commonly observed in normal embryos. The rerouting takes place at locations where cell interactions would occur in normal embryos; the timing and position of these path changes therefore suggest that neural crest migration is normally shaped by cell-cell interactions. These interactions may be direct, or might be comediated by the environment or other cells. After ablations, neural crest cells sometimes deviate into areas that are normally devoid of neural crest cells. For example, some neural crest cells enter the mesenchyme adjacent to $\mathrm{r} 3$ and $\mathrm{r} 5$, suggesting that cells can overcome the repulsive cues thought to maintain these neural crest-free zones in normal embryos. Another possibility is that the ablation of dorsal $\mathrm{r} 3$ or $\mathrm{r} 5$ may disrupt signals from the hindbrain to the adjacent mesoderm/ECM. Consistent with this, recent experiments (Golding et al., 2000) offer evidence that the neural crest-free region adjacent to $\mathrm{r} 3$ depends on the ErbB4 expression in $\mathrm{r} 3$. Our data from $\mathrm{r} 3-\mathrm{r} 4$ ablations suggest this indirect effect is not the major determinant of the rerouting after ablation, as the region lateral to $\mathrm{r} 3$ remained unpopulated. Thus, the interpretation of even strong cues may be context-dependent, suggesting that both normal and perturbed neural crest cell migration pathways are sculpted by a dynamic and interdependent set of interactions amongst neural crest and between these cells and their local environment.

This work was supported by USPHS HD15527 and a grant from the Muscular Dystrophy Foundation to M.B.F., and a BurroughsWellcome PPG (AR42671) to S.F. P.K. would like to thank the Sloan Foundation and the Burroughs-Wellcome Computational Molecular Biology Initiative at Caltech for their generous support. We are grateful to $\mathrm{H}$. McBride and D. Crotty for critical reading of the manuscript.

\section{REFERENCES}

Bilozur, M. E. and Hay, E. D. (1988). Neural crest migration in 3D extracellular-matrix utilizes laminin, fibronectin, or collagen. Dev. Biol. 125, 19-33.

Birgbauer, E., Sechrist, J., Bronner-Fraser, M. and Fraser, S. (1995). Rhombomeric origin and rostrocudal reassortment of neural crest cells revealed by intravital microscopy. Development 121, 935-945.

Buxton, P., Hunt, P., Ferretti, P. and Thorogood, P. (1997). A role for midline closure in the reestablishment of dorsoventral pattern following dorsal hindbrain ablation. Dev. Biol. 183, 150-166.

Carter, S. B. (1967). Haptotaxis and the mechanism of cell motility. Nature 213, 256-260.

Couly, G., Grapin-Botton, A., Coltey, P. and LeDouarin, N. M. (1996). The regeneration of the cephalic neural crest, a problem revisited: the regeneration of cells originate from the contralateral or from the anterior and posterior neural fold. Development 122, 3393-3407.

D'Amico, M. and Noden, D. (1983). Contributions of placodal and neural crest cells to avian cranial peripheral ganglia. Am. J. Anat. 166, 445-468..

Farlie, P. G., Kerr, R., Thomas, P., Symes, T., Minichiello, J., Hearn, C. J. and Newgreen, D. (1999). A paraxial exclusion zone creates patterned cranial neural crest cell outgrowth adjacent to rhombomeres 3 and 5. Dev. Biol. 213, 70-84.

Golding, J. P., Trainor, P., Krumlauf, R. and Gassmann, M. (2000). Defects in pathfinding by cranial neural crest cells in mice lacking the neuregulin receptor ErbB4. Nat. Cell Biol. 2, 103-109.

Graham, A., Heyman, I. and Lumsden, A. (1993). Even-numbered rhombomeres control the apoptotic elimination of neural crest cells from odd-numbered rhombomeres in the chick hindbrain. Development 119, 233245

Grapin-Botton, A., Bonnin, M. A., Ariza-McNaughton, L., Krumlauf, R. and LeDouarin, N. M. (1995). Plasticity of transposed rhombomeres: Hox gene induction is correlated with phenotypic modifications. Development 121, 2707-2721.

Hamburger, V. and Hamilton, H. L. (1951). A series of normal stages in the development of the chick embryo. J. Morph. 88, 49-92.

Hunt, P., Gulisano, M, Cook, M., Sham, M., Faiella, A, Wilkinson, D, Boncinelli, E. and Krumlauf, R. (1991). A distinct Hox code for the branchial region of the vertebrate head. Nature 353, 861-864.

Hunt, P., Ferretti, P., Krumlauf, R. and Thorogood, P. (1995). Restoration of normal Hox code and branchial arch morphogenesis after extensive deletion of hindbrain neural crest. Dev. Biol. 168, 584-597.

Hunt, P., Clarke, J. D. W., Buxton, P., Ferreti, P. and Thorogood, P. (1998). Stability and plasticity of neural crest patterning and branchial arch Hox code after extensive cephalic crest rotation. Dev. Biol. 198, 82104

Kirby, M. L., Hunt, P., Wallis, K. and Thorogood, P. (1997). Abnormal patterning of the aortic arch arteries does not evoke cardiac malformations. Dev. Dyn. 208, 34-47.

Kulesa, P. M. and Fraser, S. E. (1998). Neural crest cell dynamics revealed by time-lapse video microscopy of whole embryo chick explant cultures. Dev. Biol. 204, 327-344.

Kulesa, P. M. and Fraser, S. E. (2000). In ovo time-lapse analysis of chick hindbrain neural crest cell migration shows cell interactions during migration to the branchial arches. Development 127, 1161-1172.

LeDouarin, N. M. (1982). The Neural Crest. Cambridge University Press, Cambridge.

Lumsden, A. and Keynes, R. (1989). Segmental patterns of neuronal development in the chick hindbrain. Nature 337, 424-428.

Lumsden, A., Sprawson, N. and Graham, A. (1991). Segmental origin and migration of neural crest cells in the hindbrain region of the chick embryo. Development 113, 1281-1291.

McKee, G. and Ferguson, M. (1984). The effects of mesencephalic neural crest cell extirpation on the development of chicken embryos. J. Anat. 139, 491-512.

McGinnis, W. and Krumlauf, R. (1992). Homeobox genes and axial patterning. Cell 68, 283-302.

Noden, D. M. (1975). An analysis of the migratory behavior of avian cephalic neural crest cells. Dev. Biol. 42, 106-130.

Noden, D. M. (1983). The role of the neural crest in patterning of avian cranial skeletal, connective and muscle tissues. Dev. Biol. 96, 144-165.

Rasband, W. S. and Bright, D. S. (1995). NIH Image: A public domain image processing program for the macintosh. Microbeam Analy. Soc. J. 4, 137149 .

Saldivar, J. R., Sechrist, J. W., Krull, C. E., Ruffins, S. and BronnerFraser, M. (1997). Development 124, 2729-2739.

Scherson, T., Serbedzija, G., Fraser, S. E. and Bronner-Fraser, M. (1993). Regulative capacity of the cranial neural tube to form neural crest. Development 118, 1049-1061.

Sechrist, J., Nieto, M. A., Zamanian, R. T. and Bronner-Fraser, M. (1995). Regulative response of the cranial neural tube after neural fold ablation: spatiotemporal nature of neural crest regeneration and up-regulation of Slug. Development 121, 4103-4115.

Vaage, S. (1969). The segmentation of the primitive neural tube in chick embryos (Gallus domesticus). A morphological, histochemical and autoradiographic investigation. Ergbn. Anat. EntwGesch 41, 3-87.

Weiss, P. (1961). Guiding principles in cell locomotion and aggregation. Exp. Cell. Res. Suppl. 8, 260-281. 